\title{
Limitations of paperless on-line reporting of diagnostic bacteriology culture results
}

\author{
C Block, J Laloum, A Rajs, R Stalnikowicz, M Shapiro
}

\begin{abstract}
Aims-To estimate the extent to which microbiology laboratory results made available on a computerised reporting system do not reach their intended destination.
\end{abstract}

Methods-Prospective observational study of 180 urine cultures submitted from patients seen at the accident and emergency department of a 250 bed university affiliated general hospital. Observations were made of: telephone requests for results; whether results were noted in patients' charts; and antibiotic administration to patients sent home.

Results-Results were requested/recorded for $73 \%$ of 37 patients admitted to hospital and for only $23 \%$ of 143 patients sent home $\left(p<1 \times 10^{-7}\right)$. Overall, results were more frequently recorded for patients with positive cultures $(p=0.04)$. When determined separately for admitted and discharged groups, this association was not shown. Three of 14 culture positive patients sent home and for whom results were not recorded received inappropriate therapy; 19 culture negative patients were given antibiotics.

Conclusions-In view of the results, measures were instituted to ensure delivery of printed reports to the health care providers of patients not admitted from the accident and emergency department. Organisations operating computerised reporting systems in evolving health care settings must ensure that system design guarantees delivery of reports to all end-users. This will minimise therapeutic problems,

Department of Clinical Microbiology and

Infectious Diseases,

Hadassah University

Hospital, Mount

Scopus, Jerusalem,

Israel

C Block

J Laloum

A Rajs

M Shapiro

Emergency

Department

R Stalnikowicz

Correspondence to: Dr Colin Block, Department of Clinical Microbiology and Infectious Diseases,

Infectious Diseases,

Organisation, PO Box 12000 ,

Jerusalem 91120, Israel.

Jerusale

block@hadassah.org.il

Accepted for publication 4 June 1996 the results at ward or clinic level, for inclus in a patient's file, is not an automatic process, and observation in the field shows that it is neither universally practised nor consistent. In

For some years, printed reports have not lem. Rather, results have been made avail to the clinical services on-line through a ce computer. Inherent in this arrangement is physicians ordering tests must subsequen ensure that note is taken of the results as they tories at the two Hadassah hospitals in Jerusa-
Israel in general, even printed results are frequently transferred by hand into the file, and the original discarded (unpublished observations). Thus, it may be that some results do not reach the patient's chart, even if the physician has seen them. A system whereby printed reports are issued from the laboratory or printed automatically in ward or clinic at least ensures that physicians ordering the tests will receive all the results and have at least to decide on their distribution. In a paperless system, unless special arrangements are made and complied with, this cannot be guaranteed.

Patients seen in the accident and emergency department frequently have specimens sent to the clinical microbiology laboratory before being either discharged or admitted to hospital. Furthermore, for those discharged there is no electronic link (in the community) for access to laboratory reports. As patients do not require referral from their family physician in order to be received at the accident and emergency department, there is usually no reference in the chart to the community physician or clinic. The result of this is that printed reports are not mailed out.

This study represents an attempt to estimate the extent of this postulated problem and some of its consequences, by observing the fate of the results of urine cultures submitted from the accident and emergency department at the Hadassah Hospital on Mount Scopus, Jerusalem.

\section{Methods}

This was a prospective observational study. Specimens of urine for culture collected consecutively from 180 (not necessarily consecutive) patients seen at the accident and emergency department were entered into the study.

Microbiological examination and reporting were performed as usual, the results being entered into the hospital's central computer. Follow up of the use made of these results was carried out as follows: (1) all telephone enquiries regarding the study specimens were noted; (2) whether patients were admitted to hospital or released; (3) all patient charts were reviewed after several weeks, when the following items were noted: whether results of the culture appeared in the chart (either a printed copy or a hand-written note); whether antimicrobial therapy was initiated at the accident and emergency department; and whether infections other than urinary tract infection were considered at the time of starting such treatment. Telephone inquiries offered a measure of 
Table 1 Noting of urine culture results for specimens submitted on patients seen in the accident and emergency department, either by telephone enquiry or by observation of note in patients' charts: summary of important results

\begin{tabular}{lll}
\hline Group & Telephoned/result noted in chart (\%) & Comments \\
\hline Overall $(\mathrm{n}=180)$ & $60(33.3)$ & Ten charts missing \\
Culture positive $(\mathrm{n}=42)$ & $20(47.6)$ & Five charts missing \\
Culture negative $(\mathrm{n}=138)$ & $40(29.0)$ & Five charts missing \\
Hospitalised $(\mathrm{n}=37)$ & $27(73.0)$ & \\
Discharged $(\mathrm{n}=143)$ & $33(23.1) \dagger$ & Ten charts missing \\
\hline
\end{tabular}

${ }^{\star}$ Missing charts were included in the denominator as all telephone enquires were recorded irrespective of whether a patient's chart was located subsequently.

tTwo patients died on the day of admission.

whether results of tests ordered were being made known to physicians in the community. In this study, it was not feasible to determine whether results actually reached patient files at clinics in the community.

DEFINITIONS

A positive culture was a laboratory report of significant growth of a bacterium (by conventional criteria) and its susceptibility to antibacterial agents. A negative culture was one from which no bacterium was isolated, or from which a mixed flora was isolated in the absence of pyuria.

Comparisons between groups were evaluated using the $\chi^{2}$ test or Fisher's exact test as appropriate and $95 \%$ confidence intervals (CI).

\section{Results}

COMMUNICATION OF RESULTS

The disposition of the patients, telephone enquiries and appearance of culture results in the patients' records are shown in fig 1 . The important results are summarised in table 1 . In only about one third of all instances was there either a telephone enquiry or a record of the result in the patient's chart. Recording was significantly more frequent for hospitalised than for discharged patients $\left(\mathrm{p}<1 \times 10^{-7}(95 \%\right.$ CI 3.7-22.4)). This was also evident when hospitalised or discharged groups were evaluated separately by culture status $(p<0.005(95 \%$ CI $1.8-63.0)$ and $\mathrm{p}<0.0001(95 \%$ CI 2.6-24.0), respectively for hospitalised compared with discharged patients with positive cultures and for hospitalised compared with discharged patients with negative cultures). Significantly more enquiries were made or \& results recorded for patients with positive $\vec{\circ}$ cultures $(p=0.04(95 \%$ CI $1.0-4.8))$. These analyses included patients with missing charts (fig 1), on the grounds that all telephone calls were recorded irrespective of whether charts could be located. Statistical tests excluding the patients whose charts were missing had no effect on the significance of differences shown.

ANTIMICROBIAL TREATMENT INITIATED AT THE ACCIDENT AND EMERGENCY DEPARTMENT

Twenty seven patients with positive cultures were discharged home. Results were given by telephone for eight $(30 \%)$ of these. Five of the remaining $19(26 \%)$ patients' charts were missing. This left 14 patients who neither \& telephoned nor had their results noted in their charts. All received antimicrobial treatment; in three $(21 \%)$ cases the organism isolated was resistant to the drug prescribed.

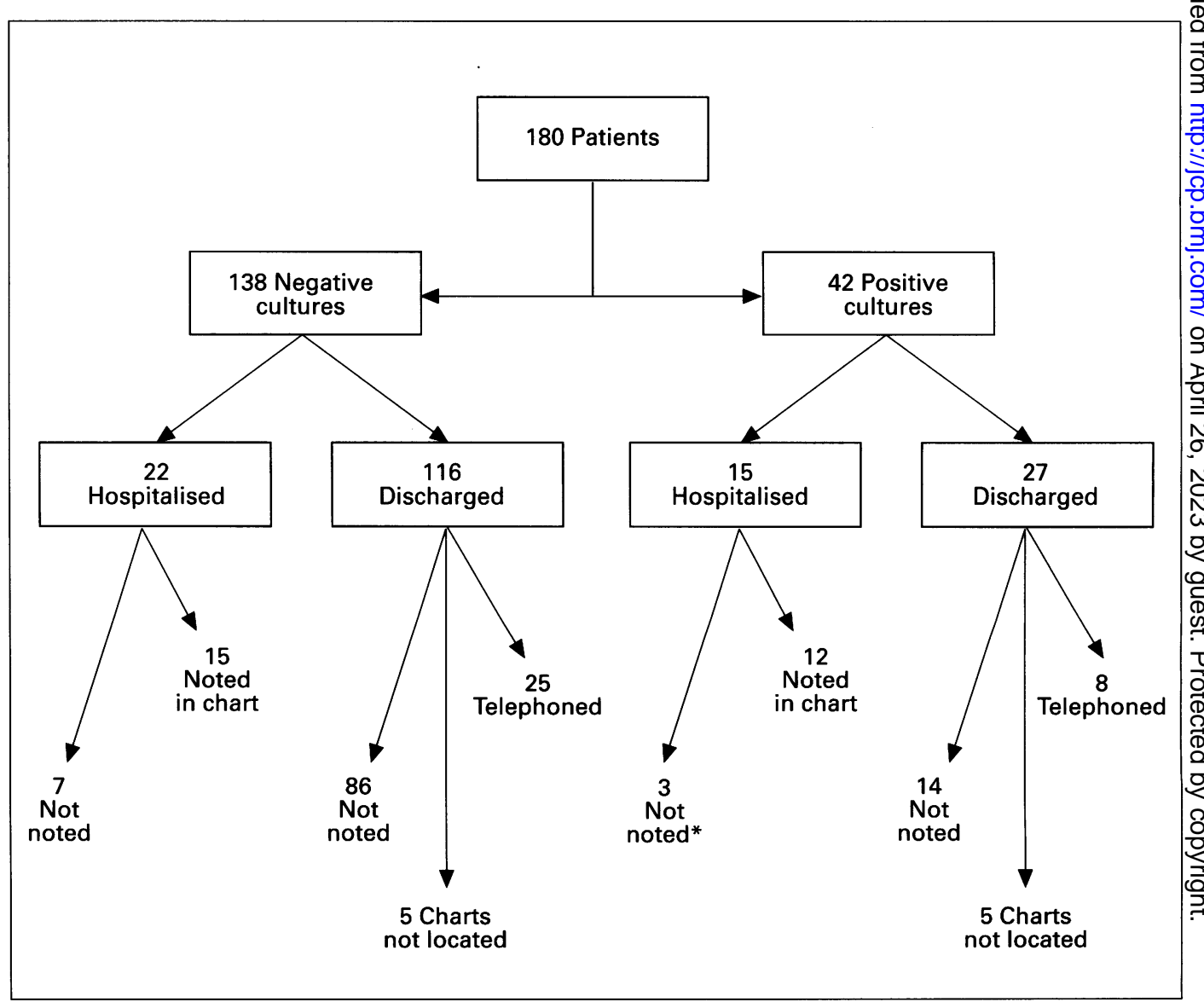

Figure 1 Disposition of 180 patients, telephone enquires and noting of culture results in patients'charts. ${ }^{\star}$ Two patients died on the day of admission. 
One hundred and sixteen patients were discharged and had negative cultures. Results were given by telephone for $25(22 \%)$ of these. The charts of five $(5 \%)$ of the other 91 were not located. The remaining 86 had no record of the results in their charts. Of these $23(27 \%)$ received antimicrobial treatment. While four had other infections, including two with upper respiratory tract infections and one each with otitis and "abdominal abscess", 19 received treatment for presumed urinary tract infection.

\section{Discussion}

Problems associated with ordering and utilisation of clinical microbiology laboratory reports are well known. ${ }^{1-5}$ Previous studies have related to physicians' general approach to ordering tests, ${ }^{1}$ the (lack of) influence of results on antibiotic prescribing, ${ }^{1-3}$ errors in reports and their effects, ${ }^{4}$ and the problems of physicians in understanding and interpreting laboratory reports. ${ }^{5}$ Only one study could be located which dealt with the recording of culture results. Howe $e t a l^{t}$ showed that, despite laboratory initiated telephone or personal communication of positive blood culture results to clinical teams, no note was made in patients' charts in 50 $(41 \%)$ of 122 of cases at 24 hours.

\section{REPORTING OF LABORATORY RESULTS}

For more than $75 \%$ of discharged patients, no attempt was made to find out and record urine culture results. While all laboratory results are continuously available on-line to hospital physicians, no such access exists for community physicians. The finding that for $27 \%$ of hospitalised patients no note was made of the results reflects not only the recognised weakness of the reporting system, but also the practice habits of the physicians. The significant differences in recording of results shown between hospitalised and discharged groups were not unexpected.

Failure to record the results in such a high proportion of cases $(70 \%$ of discharged patients) also raises the question of wastage of laboratory resources.

IMPLICATIONS FOR TREATMENT

That results were not communicated for a proportion of culture positive patients who received inappropriate antibiotics is a deviation from acceptable practice. For culture negative patients treated with antibiotics the implication is that no decision was made to stop unnecessary treatment, a flaw with cost-benefit implications as well as consequences involving adverse reactions and the promotion of emergence of antibiotic resistance.

\section{ACTION TAKEN}

When the results of this study became available, the hospital administration instituted measures to ensure delivery of printed reports to the health care providers of patients not admitted from the accident and emergency department. In addition, meetings were held with the heads of clinical departments and relevant written instructions were re-issued to all clinical staff.

\section{LIMITATIONS OF THE STUDY}

Limiting the study to urine cultures and one department raises problems for drawing general conclusions from the data. Despite this, the presence and nature of at least some of the flaws in the system were clearly shown. While no attempt was made to validate our claim that reports do not reach patients' files in their community clinics, from the nature of the computerised process it is virtually certain that this is the case.

In conclusion, organisations operating computerised reporting systems in evolving health care settings must ensure that system design promotes maximal delivery of reports to all end-users. This will help to minimise inappropriate or unnecessary treatments, limit wastage of laboratory resources, and limit risks of litigation by providing complete and accessible recording of clinical data.

The authors wish to acknowledge the advice and encouragement of the Hospital Director, Professor Jacques Michel.

1 Edwards LD, Levin S, Balagtas R, Lowe P, Landau W, Lepper MH. Ordering patterns and utilization of bacteriologic culture reports. Arch Intern Med 1973;132:678-82.

2 Campo L, Mylotte JM. Use of microbiology reports by physicians in prescribing antimicrobial agents. $A m f \mathrm{Med} S c i$ sicians in prescribin

3 Barnes MP. Influence of laboratory reports on prescribing of antimicrobials for urinary tract infection. $\mathcal{F}$ Clin Patho 1980;33:481-3

4 Howanitz PJ, Walker K, Bachner P. Quantification of errors in laboratory reports. A quality improvement study of the College of American Pathologists' Q-Probes program. Arch Pathol Lab Med 1992;116:694-700.

5 Ackerman VP, Pritchard RC, Groot Obink DJ, Bradbury R, Lee A. Consumer survey on microbiology reports. Lancet 1979;i: 199-202.

6 Howe RA, Bates CJ, Cowling P, Young N, Spencer RC. Documentation of blood culture results. $\mathcal{f}$ Clin Patho 1995;48:667-9. 